\title{
A Spatial Model to Describe Foot and Mouth Disease Dissemination ${ }^{1}$
}

N.A. MAIDANA2, Centro de Matemática, Computação e Cognição, Universidade Federal do ABC. Rua Catequese 242, 09090-400 Santo André, SP, Brasil.

H.M. YANG ${ }^{3}$, Departamento de Matemática Aplicada, Instituto de Matemática, Estatística e Computação Científica, UNICAMP, Cx.P. 6065, 13083-859 Campinas, SP, Brasil.

\begin{abstract}
In this work we propose a spatial model to analyze the foot and mouth dissemination in Mato Grosso do Sul, Brazil. The model aims to study this dissemination based on a system of partial differential reaction-diffusion equations taking into account susceptible, infected (clinical and subclinical) and removed animal subpopulations. Diffusion and advection are allowed for susceptible, subclinically infected and removed subpopulations. The traveling wave solutions of the model are searched in order to determine the speed of the disease dissemination. This wave speed is obtained as a function of the model's parameters, from which we assess the control strategies.
\end{abstract}

Keywords. Foot and mouth disease, Reaction-Diffusion Equation, Traveling waves, Wave speed.

\section{Introduction}

Foot and mouth disease (FMD) is a highly transmissible viral infection, which spreads very rapidly among animals, such as cattle, bovines, sheep and swine (very low transmission). The transmission occurs directly or indirectly by animate and inanimate vectors, and airborne route (droplets) [12].

In this work, we propose a PDE model to study the spatial dissemination of FMD. In previous works, FMD modelings considered ODE models (see Keeling and Rohani [4], chapter 4), and Individual Based models (Keeling et al. [2] and [3]). In the ODE model the dynamics of FMD was studied considering two populations, cattle and sheep, taking into account only two subpopulations, susceptible and infected. Sheep are not very competent to transmit FMD, but the coexistence with cattle can reverse this situation. In Individual Based Models, Keeling [2], [3], a random spatial dissemination of FMD was studied considering the cow population (only female subpopulation).

\footnotetext{
${ }^{1}$ FAPESP and SBMAC

${ }^{2}$ norberto.maidana@ufabc.edu.br

${ }^{3}$ hyunyang@ime.unicamp.br
} 
In our work, we consider a PDE system to describe continuous dissemination. We consider the susceptible, infected (clinical and subclinical) and recovered animals. The infected subpopulation is divided into two classes, clinical and subclinical, because it is difficult to apply control strategies in the second state.

In the next section we propose the model and study the spatially homogeneous dynamics. In section 3 we study the spatial dissemination using the traveling waves solution. Conclusion are stated in section 4 .

\section{Model for Foot and Mouth Disease (FMD)}

We present a spatial model for FMD propagation and the analysis of the corresponding spatially homogeneous model for FMD.

\subsection{Model for the spatial FMD dissemination}

We propose a spatially homogeneous model for the animal population. The population is divided into susceptible, infective (clinical and subclinical) and recovered subpopulations, $S(t), I_{s}(t), I_{c}(t)$ and $R(t)$, respectively. The total population is $N(t)=S(t)+I_{s}(t)+I_{c}(t)+R(t)$, which is allowed to vary. Let us define $\phi$ as the constant recruitment rate due to birth and migration (movement of animals among farms), and the per-capita death rate as $\mu$. The differential equation for the population irrespective of FMD is, then

$$
\frac{d N}{d t}=\phi-\mu N
$$

The infection rate per susceptible individuals is proportional to the infected subpopulation, and is given by $\beta\left(I_{s}+I_{c}\right)$. It is assumed that subclinical infected class pass through the clinical infected class at a rate $\gamma$. The clinical infected class is recovered at a rate $\epsilon$. A treatment in subclinical infected subpopulation is considered, and they pass through recovered class at a rate $\tau$. A death rate due to the sacrifice of clinical infected class is considered, and is denoted by $\delta$. Vaccination and loss of immunity are considered, and they are denoted by $\nu$ and $\Pi$, respectively.

From now on we consider the spatio-temporal dependence in the populations, e.g., $N(x, t)$ and the respective subpopulations. The diffusion in susceptible and recovered classes is denoted by $D_{1}$, while $D_{2}$ is designed for the diffusion of the subclinical infected class. The clinical infected subpopulation is considered sessile, due to sanitary control. The advection coefficients are denoted by $\lambda_{1}$ for the susceptible and recovered classes and $\lambda_{2}$ for the subclinical infected class.

Based on the above assumptions and definitions of the parameters, the spatial model (see [5], [6] for more details) is the following

$$
\begin{aligned}
\frac{\partial S}{\partial t} & =D_{1} \frac{\partial^{2} S}{\partial x^{2}}-\lambda_{1} \frac{\partial S}{\partial x}+\phi-\beta S\left(I_{s}+I_{c}\right)-\mu S-\nu S+\Pi R \\
\frac{\partial I_{s}}{\partial t} & =D_{2} \frac{\partial^{2} I_{s}}{\partial x^{2}}-\lambda_{2} \frac{\partial I_{s}}{\partial x}+\beta S\left(I_{s}+I_{c}\right)-\mu I_{s}-\gamma I_{s}-\tau I_{s}
\end{aligned}
$$




$$
\begin{aligned}
& \frac{\partial I_{c}}{\partial t}=\gamma I_{s}-(\mu+\epsilon+\delta) I_{c} \\
& \frac{\partial R}{\partial t}=D_{1} \frac{\partial^{2} R}{\partial x^{2}}-\lambda_{1} \frac{\partial R}{\partial x}+\epsilon I_{c}-\mu R+\nu S+\tau I_{s}-\Pi R .
\end{aligned}
$$

\subsection{Model for the spatially homogeneous FMD dynamics}

The spatially homogeneous model corresponding to the system $(2.1)-(2.4)$ is the following

$$
\begin{aligned}
\frac{d S}{d t} & =\phi-\beta S\left(I_{s}+I_{c}\right)-\mu S-\nu S+\Pi R \\
\frac{d I_{s}}{d t} & =\beta S\left(I_{s}+I_{c}\right)-\mu I_{s}-\gamma I_{s}-\tau I_{s} \\
\frac{d I_{c}}{d t} & =\gamma I_{s}-(\mu+\epsilon+\delta) I_{c} \\
\frac{d R}{d t} & =\epsilon I_{c}-\mu R+\nu S+\tau I_{s}-\Pi R .
\end{aligned}
$$

The system of equations $(2.5)-(2.8)$ has two steady states. The first is the disease-free equilibrium point given by

$$
P_{0}=(\hat{S}, 0,0, \hat{R}),
$$

where $\hat{S}$ and $\hat{R}$ are

$$
\hat{S}=\frac{\phi(\mu+\Pi)}{(\mu+\nu)(\mu+\Pi)-\Pi \nu}, \hat{R}=\frac{\nu \phi}{(\mu+\nu)(\mu+\Pi)-\Pi \nu},
$$

with $\hat{S}$ and $\hat{R}$ being always greater than zero.

The second one is the endemic state

$$
P_{1}=\left(S^{*}, I_{s}^{*}, I_{c}^{*}, R^{*}\right),
$$

where $S^{*}, I_{s}^{*}, I_{c}^{*}$ and $R^{*}$ are given by

$$
\begin{aligned}
& S^{*}=\frac{(\delta+\epsilon+\mu)(\gamma+\mu+\tau)}{\beta(\delta+\epsilon+\gamma+\mu)}, \quad I_{s}^{*}=\frac{\phi\left[1-\frac{1}{R_{0}}\right]}{[\mu+\gamma+\tau]-\frac{\Pi}{\mu+\Pi}\left[\frac{\epsilon \gamma}{\mu+\epsilon+\gamma}+\tau\right]}, \\
& I_{c}^{*}=\frac{\gamma I_{s}^{*}}{\mu+\epsilon+\delta}, \quad R^{*}=\frac{\left[\tau+\frac{\epsilon \gamma}{\mu+\epsilon+\delta}\right] I_{s}^{*}+\nu S^{*}}{\mu+\Pi} .
\end{aligned}
$$

Since $[\mu+\gamma+\tau]-\frac{\Pi}{\mu+\Pi}\left[\frac{\epsilon \gamma}{\mu+\epsilon+\gamma}+\tau\right]$ is always positive, a positive solution always exists for $R_{0}>1$, where

$$
R_{0}=\frac{\beta(\delta+\epsilon+\gamma+\mu)}{(\delta+\epsilon+\mu)(\gamma+\mu+\tau)} \frac{\phi(\mu+\Pi)}{(\mu+\nu)(\mu+\Pi)-\Pi \nu}
$$


is the Basic Reproductive Number. For $R_{0}<1$ the disease-free equilibrium point $P_{0}$ is locally asymptotically stable, otherwise the endemic state $P_{1}$ is stable. lished

The following Theorem, with respect to these two equilibrium points, is estab-

Theorem 2.1. The disease-free equilibrium $P_{0}$ is unique and locally asymptotically stable for $R_{0}<1$. When $R_{0}>1, P_{0}$ becomes unstable, and the endemic equilibrium $P_{1}$ appears, which is locally asymptotically stable.

Proof. See Appendix.

In order to assign values for the parameters, we consider the cows as the animal population. For the life expectancy we take $\mu^{-1}=20$ years. Considering the Mato Grosso do Sul state, we have 25000000 animals in $358158.7 \mathrm{~km}^{2}$, then we take $\frac{\phi}{\mu}=70$ per $\mathrm{km}^{2}$ [11]. We consider a latent period of 7 days and a subclinical period of 3 days (Chowell et. al [1]), then we have $\gamma^{-1}=10$ days. For the clinical onset of disease we consider $\epsilon^{-1}=5$ days [12].

We estimate the contact rate by considering the susceptibility and transmission rates of cows and sheep taken from Keeling and Rohani [4]. We consider the ODE model in Chapters 4 for cattle and sheep, and the Individual Based model in chapter 7 for cows (only female subpopulation). FMD is better transmitted by cows than sheep (ratio cow : sheep $=1.5098: 1$ ), and cows are more susceptible (ratio cow : sheep $=10.5: 1)$. Using these values we can estimate the contact rate $\beta$ for cows. Another fact that we take into account is that both models (our model and the model proposed by Keeling and Rohani [4]) consider the pseudo mass action, hence, the contact parameter must be scaled by the population size. We obtain $\beta=0.00825078$. We do not consider any control.

Figure 1 shows the dynamics of FMD disease. We can see the peak of the infected subpopulation. The subclinical infected peak is greater than the clinical one, and the subclinical infected burst occurs previously in time. The proportion of clinical infected cows (see Figure 1) is closer to the proportion obtained for cattle in the ODE model considering cattle and sheep proposed by Keeling and Rohane [4] to study the Cuimbra FMD epidemic in 2001. Notice that as the cattle population, cows are more susceptible and FMD is better transmitted by cows than sheep, then we expect that the proportion of infected cattle is similar to that of infected cows.

\section{Traveling Waves Solution}

In this section we study the geographic propagation of FMD using the same method applied to describe the dissemination of rabies among foxes [8], [7], that is, we determine the minimum wave speed connecting the disease-free equilibrium point and the endemic state. The solution corresponding to the minimum wave speed of the system of equations $(2.1)-(2.4)$ describes the observed biological waves, see Volpert [10] and Sandstede [9].

The traveling waves solution, when it exists, can be set in the usual form [6]

$$
\left(s(x, t), i_{s}(x, t), i_{c}(x, t), r(x, t)\right)=\left(s(z), i_{s}(z), i_{c}(z), r(z)\right),
$$




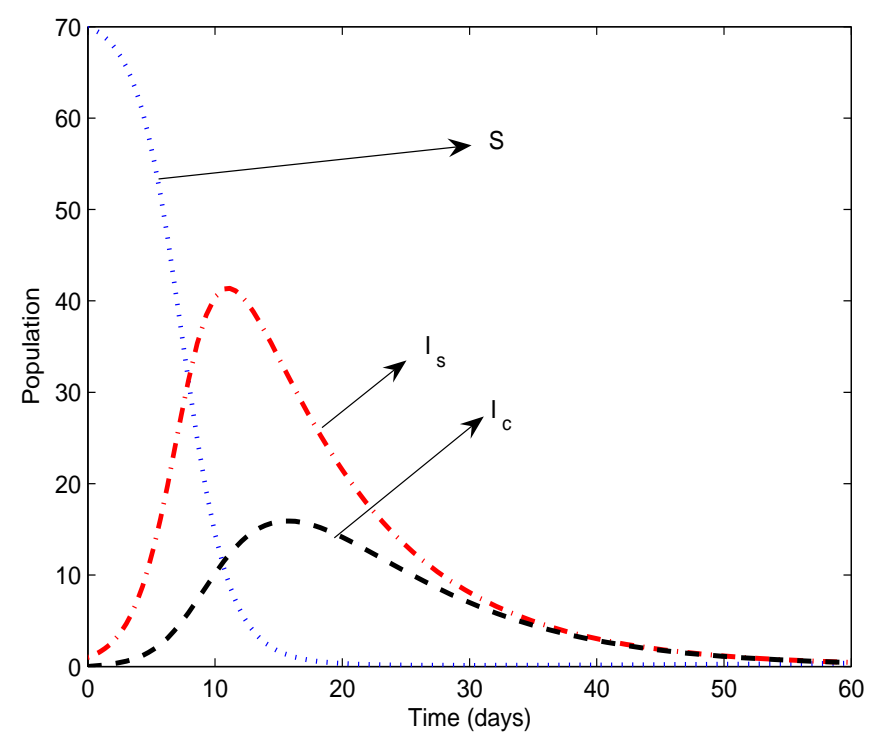

Figure 1: Graph of the numbers of susceptible, subclinical and clinical infected subpopulations. We can observe the epidemic burst, after the introduction of one infected animal in subclinical condition. The initial values are $S(0)=70, I_{s}(0)=1$, $I_{c}(0)=0$ and $R(0)=0$. The subclinical infected peak is high, 40 infected cows of 70 cows, the total susceptible population in the disease-free equilibrium state. The peak for infected clinical is lower, 15 individuals.

where $z=x+$ ct. In this new variable, the equations (2.1) - (2.4) are transformed into

$$
\begin{aligned}
c \frac{d s}{d z} & =D_{1} \frac{d^{2} s}{d z^{2}}-\lambda_{1} \frac{d s}{d z}+\phi-\beta s\left(i_{s}+i_{c}\right)-\mu s-\nu s+\Pi r \\
c \frac{d i_{s}}{d z} & =D_{2} \frac{d^{2} i_{s}}{d z^{2}}-\lambda_{2} \frac{d i_{s}}{d z}+\beta s\left(i_{s}+i_{c}\right)-\mu i_{s}-\gamma i_{s}-\tau i_{s} \\
c \frac{d i_{c}}{d z} & =\gamma i_{s}-(\mu+\epsilon+\delta) i_{c} \\
c \frac{d r}{d z} & =D_{1} \frac{d^{2} r}{d z^{2}}-\lambda_{1} \frac{d r}{d z}+\epsilon i_{c}-\mu r+\nu s+\tau i_{s}-\Pi r .
\end{aligned}
$$

Defining the variables $u_{1}=\frac{d s}{d z}, u_{2}=\frac{d i_{s}}{d z}$, and $u_{3}=\frac{d r}{d z}$, the corresponding first order ordinary differential equations with respect to variable $z$ of the system (3.2) $-(3.5)$ is 


$$
\begin{aligned}
\frac{d s}{d z} & =u_{1} \\
\frac{d u_{1}}{d z} & =\frac{1}{D_{1}}\left[\left(c+\lambda_{1}\right) u_{1}-\phi+\beta s\left(i_{s}+i_{c}\right)+\mu s+\nu s-\Pi r\right] \\
\frac{d i_{s}}{d z} & =u_{2} \\
\frac{d u_{2}}{d z} & =\frac{1}{D_{2}}\left[\left(c+\lambda_{2}\right) u_{2}-\beta s\left(i_{s}+i_{c}\right)+\mu i_{s}+\gamma i_{s}+\tau i_{s}\right] \\
\frac{d i_{c}}{d z} & =\frac{1}{c}\left[\gamma i_{s}-(\mu+\epsilon+\delta) i_{c}\right] \\
\frac{d r}{d z} & =u_{3} \\
\frac{d u_{3}}{d z} & =\frac{1}{D_{1}}\left[\left(c+\lambda_{1}\right) u_{3}-\epsilon i_{c}+\mu r-\nu s-\tau i_{s}+\Pi r\right],
\end{aligned}
$$

where the boundary conditions are

$$
\lim _{z \rightarrow-\infty}\left(s_{a}(z), u_{1}(z), i_{a}(z), u_{2}(z), i_{v}(z), n_{a}(z), u_{3}(z)\right)=(\hat{S}, 0,0,0,0, \hat{R}, 0)
$$

and

$$
\lim _{z \rightarrow \infty}\left(s_{a}(z), u_{1}(z), i_{a}(z), u_{2}(z), i_{v}(z), n_{a}(z), u_{3}(z)\right)=\left(S^{*}, 0, I_{s}^{*}, 0, I_{c}^{*}, R^{*}, 0\right) .
$$

The zeros in both equilibrium points deserve some comments. The three zeros in the second equilibrium point correspond to derivatives of the subpopulations $s, i_{s}$ and $r$. However, the first equilibrium point has two more zeros corresponding to infectious populations regarding to clinical and subclinical cases, which must not assume negative values. Due to this constraint, we impose to the linear system solutions that must not oscillate, i.e., the eigenvalues corresponding to this equilibrium point must assume real values.

The roots of the characteristic polynomial regarding the linear system at the equilibrium point $\left(s_{a}, u_{1}, i_{a}, u_{2}, i_{v}, n_{a}, u_{3}\right)=(\hat{S}, 0,0,0,0, \hat{R}, 0)$ are the roots of the polynomials $Q_{1}(\lambda), Q_{2}(\lambda)$ and $P(\lambda)$, where

$$
\begin{gathered}
Q_{1}(\lambda)=D_{1} \lambda^{2}-\left(c+\lambda_{1}\right) \lambda-\mu, \\
Q_{2}(\lambda)=D_{1} \lambda^{2}-\left(c+\lambda_{1}\right) \lambda-(\mu+\nu+\Pi)
\end{gathered}
$$

and

$$
P(\lambda)=D_{2} \lambda^{3}+A \lambda^{2}+B \lambda+C,
$$


which has the coefficients

$$
\begin{aligned}
& A=-\left(c+\lambda_{2}\right)+\frac{(\delta+\epsilon+\mu) D_{2}}{c}, \\
& B=-(\tau+\gamma+\mu)+\beta \hat{S}-\frac{(\delta+\epsilon+\mu)\left(c+\lambda_{2}\right)}{c}, \\
& C=\frac{(\delta+\epsilon+\mu)(\gamma+\mu+\tau)}{c}\left(R_{0}-1\right),
\end{aligned}
$$

with $R_{0}$ being given by (2.9). Polynomials $Q_{1}(\lambda)$ and $Q_{2}(\lambda)$ always have real roots. Then the polynomial $P(\lambda)$ must determine the conditions for the existence of the minimum speed, that is, the eigenvalues are real. The minimum velocity is determined by the condition that the polynomial evaluated at the unique local minimum, $\lambda_{+}$, must be zero, that is, $P\left(\lambda_{+}\right)=0$, where

$$
\lambda_{+}=\frac{1}{3 D_{2}}\left\{-A+\sqrt{A^{2}-3 B}\right\} .
$$

In Figure 2 the wave speed varying as a function of the diffusion coefficient is shown. We can observe the importance of the direct transmission in the disease spread. This is a consequence of the high contact rate. For a diffusion coefficient value of $100 \mathrm{~m}^{2}$ per day, the wave speed is $457.417 \mathrm{~m}$ per day.

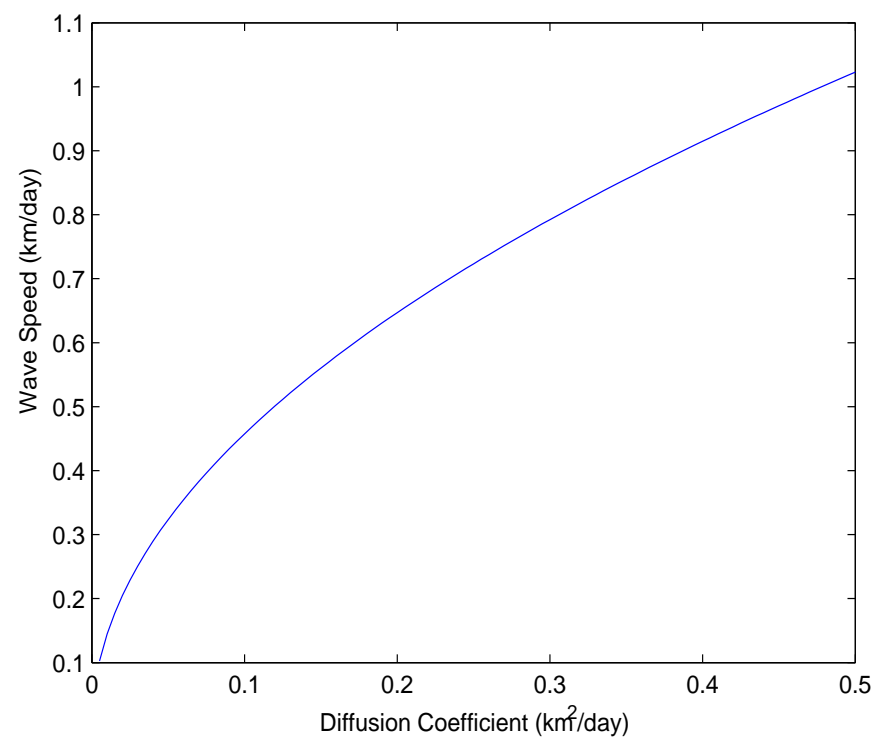

Figure 2: Graph of the wave speed as a function of the diffusion coefficient for the parameters listed in the previous section. 


\section{Conclusion}

In this paper we developed a spatial propagation model to understand the dissemination of the FMD. For the spatially homogeneous dynamics we determined, in non dimensional parameters, the threshold value

$$
R_{0}=\frac{\beta(\delta+\epsilon+\gamma+\mu)}{(\delta+\epsilon+\mu)(\gamma+\mu+\tau)} \frac{\phi(\mu+\Pi)}{(\mu+\nu)(\mu+\Pi)-\Pi \nu} .
$$

When $R_{0}$ is greater than one, the endemic state of the disease exists. We study the wave speed for the traveling waves connecting this endemic point with the disease-free equilibrium point. An equation for the minimum speed was determined as a function of the parameters of the model and the threshold $R_{0}$.

We use this equation to determine the wave speed as a function of the diffusion coefficient. Foot and mouth disease spreads by direct contact between animals and indirectly by vectors (animate or inanimate). We can observe that the direct transmission must be considered as important route in the disease spreading. This is a consequence of the high value of the direct contact rate for this disease.

\section{A Stability Analysis}

In this section we analyze the stability of the equilibrium points.

\subsection{Stability of $P_{0}=(\hat{S}, 0,0, \hat{R})$}

By linearizing the system $(2.5)-(2.8)$ at the equilibrium point $P_{0}$ we obtain the jacobian matrix

$$
J\left(P_{0}\right)=\left(\begin{array}{cccc}
-\mu-\nu & -\frac{\beta \phi(\mu+\phi)}{\mu(\mu+\phi+\nu)} & -\frac{\beta \phi(\mu+\phi)}{\mu(\mu+\phi+\nu)} & \pi \\
0 & -\mu-\gamma-\tau+\frac{\beta \phi(\mu+\phi)}{\mu(\mu+\phi+\nu)} & \frac{\beta \phi(\mu+\phi)}{\mu(\mu+\phi+\nu)} & 0 \\
0 & \gamma & -\delta-\epsilon-\mu & 0 \\
\nu & \tau & \epsilon & -\mu-\pi
\end{array}\right) .
$$

The eigenvalues are $-\mu,-\mu-\nu-\pi$, and the roots of the polynomial

$$
p_{1}(\lambda)=\lambda^{2}+[\delta+\epsilon+\gamma+2 \mu+\tau-\beta \hat{S}] \lambda+(\epsilon+\delta+\mu)(\tau+\gamma+\mu)\left(1-R_{0}\right) .
$$

It is easy to check for $R_{0}<1$, that

$$
\begin{gathered}
\delta+\epsilon+\gamma+2 \mu+\tau-\beta \hat{S}>\delta+\epsilon+\gamma+2 \mu+\tau-\frac{(\delta+\epsilon+\mu)(\gamma+\mu+\tau)}{\delta+\epsilon+\gamma+\mu}> \\
(\delta+\epsilon+\gamma+2 \mu+\tau)(\delta+\epsilon+\gamma+\mu)-(\delta+\epsilon+\mu)(\gamma+\mu+\tau)>0 .
\end{gathered}
$$

Then, all coefficients of polynomial $p_{1}(\lambda)$ are positive if and only if $R_{0}<1$. Therefore, $P_{0}$ is locally asymptotically stable if $R_{0}<1$ and is unstable if $R_{0}>1$. 


\subsection{Stability of $P_{1}=\left(S^{*}, I_{s}^{*}, I_{c}^{*}, R^{*}\right)$}

The non trivial equilibrium point $P_{1}$ exists if and only if $R_{0}>1$. This condition implies that $S^{*} \geq 0, I_{s}^{*} \geq 0, I_{c}^{*} \geq 0$ and $R^{*} \geq 0$. We use these facts in the proof of this Theorem. The local stability is determined by the roots of $\operatorname{Det}\left(\lambda I d-J\left(P_{1}\right)\right)$, where $J\left(P_{1}\right)$ is given by

$$
J\left(P^{1}\right)=\left(\begin{array}{cccc}
-\left(I_{s}^{*}+I_{c}^{*}\right) \beta-\mu-\nu & -S^{*} \beta & -S^{*} \beta & \pi \\
-\left(I_{s}^{*}+I_{c}^{*}\right) \beta & S^{*} \beta-\gamma-\mu-\tau & -S^{*} \beta & 0 \\
0 & -\gamma & -\delta-\epsilon-\mu & 0 \\
\nu & \tau & \epsilon & -\mu-\pi
\end{array}\right)
$$

where $I d$ is a $4 \times 4$ identity matrix. For simplicity, we consider the case without loss of immunity $(\pi=0)$, which is due to an effective vaccine.

The eigenvalues at the point $P_{1}(\pi=0)$ are $-\mu$, for instance, see that the fourth column of $J\left(P_{1}(\pi=0)\right)$ has three zeros, and the other roots are given by the polynomial

$$
p_{2}(\lambda)=\lambda^{3}+a_{2} \lambda^{2}+a_{1} \lambda+a_{0},
$$

where

$$
\begin{aligned}
& a_{2}=\beta\left(I_{c}^{*}+I_{s}^{*}\right)-\beta S^{*}+\gamma+\delta+\epsilon+3 \mu+\nu+\tau, \\
& a_{1}=-\beta S^{*}(\gamma+\epsilon+\delta+2 \mu+\nu)+\gamma \delta+\gamma \epsilon+2 \gamma \mu+2 \delta \mu+2 \delta \epsilon+3 \mu^{2}+\gamma \nu+\delta \nu+\epsilon \nu+ \\
& +2 \mu \nu+\delta \tau+\epsilon \tau+2 \mu \tau+\nu \tau+\beta\left(I_{c}^{*}+I_{s}^{*}\right)(\gamma+\delta+\epsilon+2 \mu+\tau), \\
& a_{0}=\beta\left(I_{c}^{*}+I_{s}^{*}\right)(\delta+\epsilon+\mu)(\gamma+\mu+\tau) .
\end{aligned}
$$

From the Routh-Hurwitz criteria, it follows that all eigenvalues have negative real part if and only if, $a_{2}>0, a_{0}>0$ and $a_{2} a_{1}>a_{0}$. Trivially, we have $a_{0}>0$.

$$
\begin{aligned}
& \text { Using that } S^{*}=\frac{(\gamma+\mu+\tau)(\mu+\epsilon+\delta)}{\beta(\mu+\epsilon+\delta+\gamma)}, a_{1} \text { and } a_{2} \text { become } \\
& a_{1}=(\mu+\epsilon+\delta)(\mu+\nu)+\frac{(\mu+\nu)(\mu+\gamma+\tau) \gamma}{\mu+\epsilon+\delta+\gamma}+(\gamma+\delta+\epsilon+2 \mu+\tau) \beta\left(I_{c}^{*}+I_{c}^{*}\right)>0 \\
& \text { and } \\
& a_{2}=\frac{(\mu+\gamma+\tau) \gamma}{\mu+\epsilon+\delta+\gamma}+\beta\left(I_{c}^{*}+I_{s}^{*}\right)+\delta+\epsilon+2 \mu+\nu>0 .
\end{aligned}
$$

Finally, we have

$$
a_{1} a_{2}>\left[(\gamma+\mu+\tau) \beta\left(I_{c}^{*}+I_{s}^{*}\right)\right][\delta+\epsilon+\mu]=a_{0} .
$$

Then the equilibrium point $P_{1}$ is locally asymptotically stable if and only if $R_{0}>1$. 
Resumo. Neste trabalho propomos um modelo espacial para estudar a propagação de febre aftosa no Estado de Mato Grosso do Sul, Brasil. O objetivo é o estudo da disseminação por meio de um sistema de equações diferenciais parcias de reação difusão considerando subpopulações de animais suscetíveis, infectados (clínicos e subclínicos) e recuperados. Estudamos as soluções onda viajantes para determinar a velocidade da disseminação da doença. Esta velocidade é determinada como função dos parâmetros do modelo a fim de estabelecer estratégias de controle.

\section{References}

[1] G. Chowell, A.L. Rivas, N.W. Hengartner, J.M. Hyman, C. Castillo-Chavez, Critical response to post-outbreak vaccination against foot-and-mouth disease. Modeling The Dynamics of Human Diseases: Emerging Paradigms and Challenges. AMS Contemporary Mathematics Series, vol. 410, pp. 73-87, 2006.

[2] M.J. Keeling, M.E.J. Woolhouse, D.J. Shaw, B.T. Matthews, Dynamic of the 2001 UK foot and mouth epidemic: stochastic dispersal in heterogeneous landscape, Science, 294 (2001), 813-817.

[3] M.J. Keeling, M.E.J. Woolhouse, R.M. May, G. Daives, B.T. Grenfell, Modelling vaccinations strategies against foot-and-mouth disease, Nature, 421 (2003), 136-142.

[4] M.J. Keeling, P. Rohani, Modeling Infectious Diseases in Human and Animals, Priceton University Press, 2008.

[5] N.A. Maidana, H.M. Yang, A spatial model to describe foot and mouth disease dissemination, Anais do XXXII CNMAC, Cuiabá, Mato Grosso, 2010.

[6] J.D. Murray, "Mathematical Biology", Springer, Berlin, 2002.

[7] J.D. Murray, W.L. Seward, On the spatial spread of rabies among foxes with immunity, J. Theor. Biol., 156 (1992), 327-348.

[8] J.D. Murray, F.R.S. Stanley, D.L. Brown, On the spatial spread of rabies among foxes, Proc. R. Soc. Lond., B229 (1986), 111-150.

[9] B. Sandstede, Stability of traveling waves, in "Handbook of Dynamical System II" (B. Fiedler, ed.), pp. 983-1059, Elsevier, Amsterdam, 2002.

[10] A.I. Volpert, V.A. Volpert, "Traveling Waves Solutions of Parabolic System", American Mathematical Society, Providence, RI, 1994.

[11] http://www.embrapa.br/

[12] http://www.oie.int/eng/maladies/fiches/a_A010.HTM 